\title{
Seasonal Dependence of SC-Field in Middle and Low Latitudes*
}

\author{
By Rikio MAedA**, Naoshi Fukushima and Takesi Nagata \\ Geophysical Institute, University of Tokyo \\ (Read May 25, 1962; Received Dec. 30, 1964)
}

\begin{abstract}
An analysis of world-wide geomagnetic field-change for eight SC's during IGY indicates that the uniform northward external magnetic field for SC in middle and low latitudes shows a systematic seasonal dependence, namely, the uniform external magnetic field inclines towards the sunlit side in the summer hemisphere, and towards the night side in winter. This systematic seasonal dependence of the SC-field may be explained as due to the geometrical relation between the incident direction of the solar wind and the geomagnetic dipole axis.
\end{abstract}

\section{Introduction}

The behaviour of sudden commencements of magnetic storms (SC) has recently been examined in detail with the aid of world-wide data during the International Geophysical Year, especially for the purpose of studying the electromagnetic induction within the earth (Maeda et al., 1965). While the analysis was being performed, a systematic seasonal dependence of SC in middle and low latitudes was found.

For convenience, the disturbance field for $\mathrm{SC}$, denoted by $D(S C)$, is separated into two parts: i.e.,

$$
D(S C)=D s t(S C)+D S(S C) .
$$

When a great number of SC's occurring in all seasons are averaged, the $\operatorname{Dst}(\mathrm{SC})$ currentsystem consists of an eastward electric current parallel to the geomagnetic latitude circle. The electric current intensity is nearly proportional to the cosine of the geomagnetic latitude in middle and low latitudes, except just on and near the geomagnetic equator, where an equatorial enhancement is observed (Sugiura, 1953; Obayashi and Jacobs, 1957). Most of the $\operatorname{Dst}(S C)$-field is attributable to a northward external magnetic field parallel to the geomag. netic dipole axis. On the other hand, most of the electric currents for $D S(S C)$ are concentrated in the high-latitude regions, revealing a complicated feature of SC in high latitudes, as studied by Oguti (1956), Sato (1961, 1962), Sano (1962), Matsushita (1962), and others. The current-system for SC*, the reverse impulse which precedes the main SC, is shown to be expressed by a current flow in the reverse direction to that in the developed stage of $\mathrm{SC}$ in high latitudes (Nagata, 1952; Nagata and Abe, 1955).

\footnotetext{
* Contribution from Division of Geomagnetism and Planetary Physics, University of Tokyo, Series II, No. 186.

** Now at the Radio Research Laboratories, Kokubunji, Tokyo.
} 
In the present paper, the electric current-system for the developed stage of SC is examined in detail for eight selected SC's. The seasonal dependence of the electric currentsystem in middle and low latitudes is discussed, and the current pattern in high latitudes is not dealt with.

\section{Data used in the present analysis}

Four SC's each for the summer and winter seasons during IGY are analyzed. The times of these selected SC's and of their developed stages, and the magnitude of SC in $H$, denoted by $\Delta H$, at three low-latitude stations are summarized in Table I. The observatories,

Table I. The SC's used for the present analysis.

\begin{tabular}{|c|c|c|c|c|c|c|c|c|c|c|c|}
\hline \multirow{2}{*}{$\begin{array}{c}\text { Event } \\
\text { No. } \\
1\end{array}$} & \multicolumn{4}{|c|}{ Time of SC in U.T. $\left(T_{0}\right)$} & \multicolumn{2}{|c|}{$T_{1}$} & \multirow{2}{*}{$\begin{array}{c}\begin{array}{c}\text { Rang } \\
\text { Kaki- } \\
\text { oka }\end{array} \\
18 \gamma\end{array}$} & \multirow{2}{*}{$\begin{array}{l}\begin{array}{c}\text { of } \Delta H \\
\text { San } \\
\text { Juan }\end{array} \\
14 \gamma\end{array}$} & \multirow{2}{*}{$\begin{array}{c}\begin{array}{c}\text { C) at } \\
\text { Huan- } \\
\text { cayo }\end{array} \\
40 \gamma\end{array}$} & \multirow{2}{*}{$\frac{(K p)_{\mathrm{SC}}}{3_{0}}$} & \multirow{2}{*}{$\frac{(K p)_{\max }}{4_{0}}$} \\
\hline & 1957 & July $16^{d}$ & $07^{\mathrm{h}}$ & $14^{\mathrm{m}}$ & $07^{\mathrm{b}}$ & $19^{\mathrm{m}}$ & & & & & \\
\hline 2 & 1958 & Feb. 11 & 01 & 25 & 01 & 28 & 36 & 52 & 167 & $9_{0}$ & $9_{0}$ \\
\hline 3 & 1958 & July 08 & 07 & 48 & 07 & 51 & 110 & 52 & 97 & $7-$ & $9_{0}$ \\
\hline 4 & 1958 & July 21 & 16 & 38 & 16 & 40 & 47 & 31 & 178 & $7-$ & $7+$ \\
\hline 5 & 1958 & Aug. 17 & 06 & 22 & 06 & 24 & 41 & 50 & 90 & $6_{0}$ & $7+$ \\
\hline 6 & 1958 & Sep. 25 & 04 & 08 & 04 & 13 & 37 & 23 & 32 & $6_{0}$ & 70 \\
\hline 7 & 1958 & Dec. 13 & 00 & 01 & 00 & 03 & 16 & 18 & 45 & $5+$ & $6_{0}$ \\
\hline 8 & 1958 & Dec. 17 & 18 & 17 & 18 & 18 & 37 & 24 & 117 & $6+$ & $7_{0}$ \\
\hline
\end{tabular}

Legend: $T_{1}$ : Time in U.T. of the developed stage of SC.

$(K p)_{\mathrm{SC}}: K p$-index during the 3-hour interval, in which SC took place.

$(K p)_{\max }$ : Maximum $K p$ value during the magnetic storm following SC.

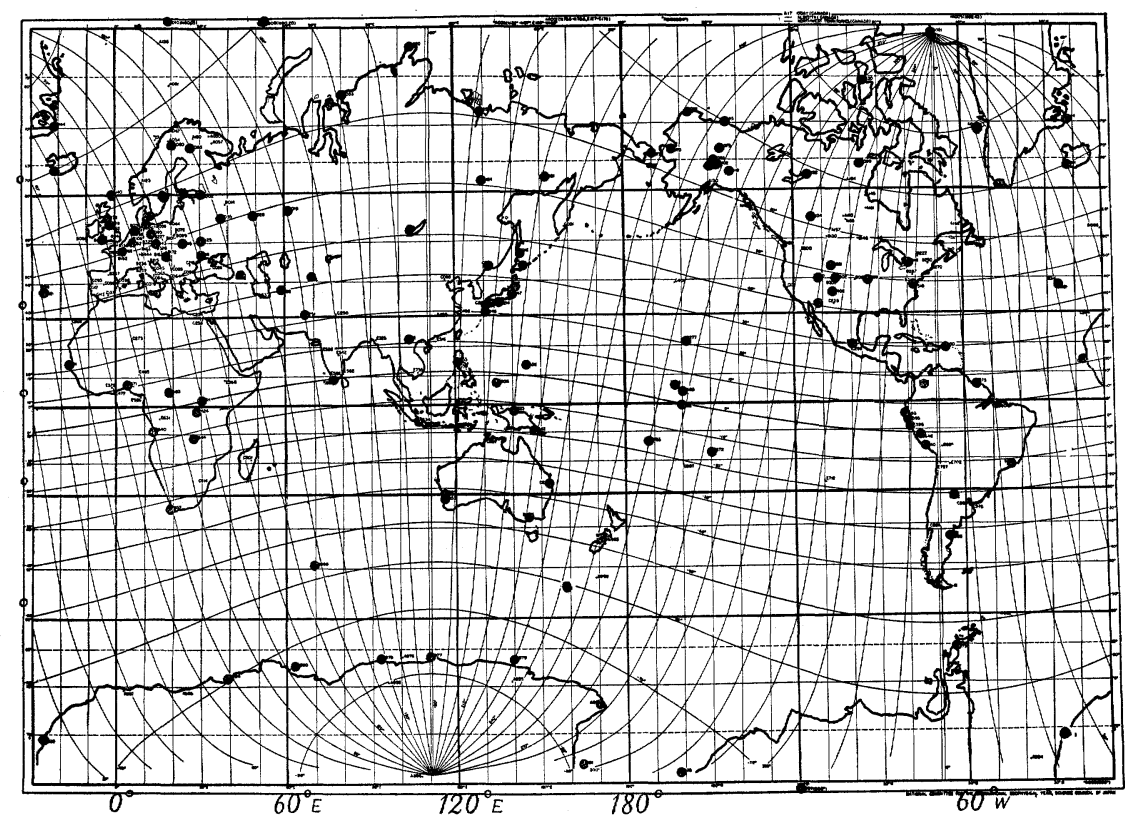

Fig. 1. Distribution of magnetic observatories, whose data are used in the present analysis. 
whose data are utilized in this study, are distributed over the world as shown in Fig. 1. The total number of stations amounts to 126 (31 stations in the arctic region, 26 in the north subauroral region, 22 in the north minauroral region, 11 in the northern equatorial zone, 15 in the southern equatorial zone, 6 in the south minauroral region, 3 in the south subauroral region, and 12 in the antarctic region).

\section{Overhead current-system for SC's}

The overhead current-systems are drawn for the developed SC-field (at time $T_{1}$ ), for each event given in Table I. Four examples of the current-systems in the northern hemisphere are shown in Fig. 2.
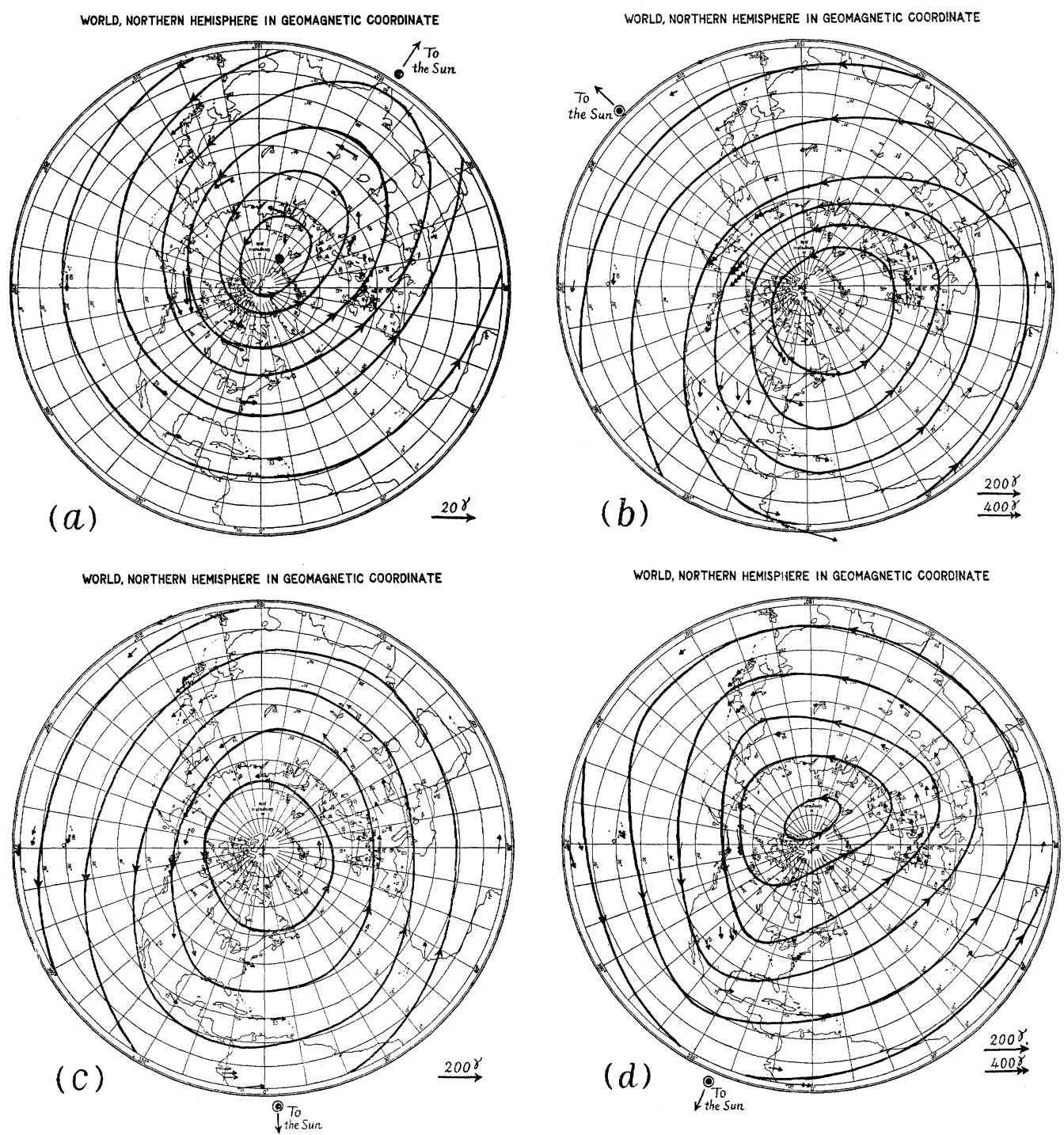

Fig. 2. Overhead current-system for the developed stage of SC, (a) 1957 July $16,07 \mathrm{~h} 19 \mathrm{~m}$; (b) 1958 February 11, 01h 28m; (c) 1958 July 21, 16h 40m; (d) 1958 December 17, 18h 18m U.T. 
The current pattern is complicated in the polar region, while it is rather simple in the middle and low latitudes. The electric current flow in the middle and low latitudes is not exactly parallel to the geomagnetic latitude circle in each case. This problem is discussed quantitatively in the next section.

\section{Spherical harmonic analysis of the $D(S C)$-field}

Based on the data in the middle and low latitudes, the $D(S C)$-field is expanded into a spherical harmonic series. The magnetic potential $W$ is given by

$$
\begin{aligned}
W & =C+R \sum_{n=1}^{\infty}\left[\left(\frac{r}{R}\right)^{n} \sum_{m=0}^{n}\left\{g_{n}{ }^{m(e)} \cos m \lambda+h_{n}{ }^{m(e)} \sin m \lambda\right\} P_{n}{ }^{m}(\cos \theta)\right. \\
& \left.+\left(\frac{R}{r}\right)^{n+1} \sum_{m=0}^{n}\left\{g_{n}{ }^{m(i)} \cos m \lambda+h_{n}{ }^{m(i)} \sin m \lambda\right\} P_{n}{ }^{m}(\cos \theta)\right]
\end{aligned}
$$

referring to geomagnetic coordinates, with customary notations in the spherical harmonic expansion. In the middle and low latitudes, the $D(S C)$-field on the earth's surface $r=R$ is well approximated by

$$
\begin{gathered}
X=-\left\{g_{1}{ }^{(e)}+g_{1}{ }^{0(i)}\right\} \sin \theta+\left[\left\{g_{1}{ }^{1(e)}+g_{1}{ }^{1(i)}\right\} \cos \lambda+\left\{h_{1}{ }^{1(e)}+h_{1}{ }^{1(i)}\right\} \sin \lambda\right] \cos \theta \\
\equiv-g_{1}{ }^{0} \sin \theta+\left[g_{1}{ }^{1} \cos \lambda+h_{1}{ }^{1} \sin \lambda\right] \cos \theta, \\
Y=\left\{g_{1}{ }^{1(e)}+g_{1}{ }^{1(i)}\right\} \sin \lambda-\left\{h_{1}{ }^{1(e)}+h_{1}{ }^{1(i)}\right\} \cos \lambda \equiv g_{1}{ }^{1} \sin \lambda-h_{1}{ }^{1} \cos \lambda .
\end{gathered}
$$

\begin{tabular}{|c|c|c|c|c|c|c|c|c|}
\hline $\begin{array}{l}\text { Event } \\
\text { No. }\end{array}$ & $\begin{array}{c}\text { Spherical } \\
g_{1}{ }^{0}\end{array}$ & $\begin{array}{l}\text { harmonic } \\
g_{1}{ }^{1}\end{array}$ & $\underset{h_{1}{ }^{1}}{\text { coefficients }}$ & $\varphi_{0}$ & $\lambda_{0}$ & $\varphi_{s}$ & $\lambda_{s}$ & $\lambda_{0}-\lambda_{s}$ \\
\hline 1 & $-32.6 \gamma$ & $7: 0 \gamma$ & $-3.8 \gamma$ & $76^{\circ} .2$ & $151^{\circ} .2$ & $12^{\circ} .5$ & $142^{\circ} .1$ & $9^{\circ} .4$ \\
\hline 2 & -106.8 & -28.4 & -25.6 & 70.3 & 42.0 & -21.7 & 231.3 & -189.3 \\
\hline 3 & -92.1 & 15.8 & -2.9 & 80.1 & 169.4 & 14.6 & 135.5 & 33.9 \\
\hline 4 & -86.8 & -23.7 & -4.5 & 74.5 & 10.7 & 32.1 & 0.5 & 10.2 \\
\hline 5 & -50.2 & 7.2 & -1.9 & 81.6 & 165.2 & 3.4 & 154.2 & 11.0 \\
\hline 6 & -47.5 & -10.5 & -1.8 & 77.4 & 9.7 & -10.9 & 187.2 & -177.5 \\
\hline 7 & -32.5 & 3.7 & -7.2 & 76.0 & 117.2 & -26.7 & 254.5 & -137.3 \\
\hline 8 & -68.3 & 11.5 & -0.4 & 80.4 & 178.0 & -12.9 & 336.5 & -158.5 \\
\hline
\end{tabular}

The coefficients obtained in the actual analysis are shown in Table II.

Table II. Spherical harmonic analysis of $D(S C)$-field in low latitudes.

Legend: $\left(\varphi_{0}, \lambda_{0}\right):$ Geomagnetic coordinates of the point in the northern hemisphere, where the axis through the earth's centre parallel to the equatorial $\operatorname{Dst}(S C)$-field intersects the earth surface.

$\left(\varphi_{s}, \lambda_{s}\right)$ : Geomagnetic coordinates of the subsolar point at the time of the developed stage of SC.

\section{Seasonal dependence of the $\operatorname{Dst}(\mathrm{SC})$-axis}

From the three coefficients, $g_{1}{ }^{0}, g_{1}{ }^{1}$ and $h_{1}{ }^{1}$, the axis of the uniform external magnetic field at the time of an SC can be determined. In Table II, $\varphi_{0}$ and $\lambda_{0}$ denote the geomagnetic latitude and longitude of the point where the direction vector of the SC uniform external magnetic field through the earth's centre intersects the earth surface in the northern hemisphere. The point $\left(\varphi_{0}, \lambda_{0}\right)$ will be referred to, hereafter, simply as the $D s t(S C)$-axis pole. $\varphi_{s}$ and $\lambda_{s}$ in Table II indicate respectively the geomagnetic latitude and longitude of the subsolar 
point at developed stage time of the SC in question.

Figs. 3 and 4 show that the $\operatorname{Dst}(\mathrm{SC})$ axis inclines towards the sunlit side in the summer hemisphere, because $\left|\lambda_{0}-\lambda_{s}\right|$ $<90^{\circ}$ when the subsolar point is in the northern hemisphere $\left(\varphi_{s}>0\right)$. The $D s t$ $(S C)$-axis is shown to incline towards the night side in winter, on the other hand, because $90^{\circ}<\left|\lambda_{0}-\lambda_{s}\right|<180^{\circ}$ when $\varphi_{s}<0$.

As mentioned before, the $\operatorname{Dst}(S C)$ field in low latitudes can be expressed by a northward external magnetic field parallel to the geomagnetic dipole axis, for the average of a great number of magnetic storms which take place throughout the year. In the present analysis, it is illustrated that the $\operatorname{Dst}(S C)$ axis shows a systematic dependence on the season. The observed relation between $\varphi_{0}$ and $\varphi_{s}$ may be approximated by the dotted line in Fig. 3, which is a theoretical curve derived according to the following consideration.

\section{Image dipole in the solar stream and its magnetic effect on the earth}

It is now widely believed that $\mathrm{SC}$ is caused by an intense solar plasma stream arriving at the vicinity of the earth. Chapman and Ferraro (1931, 1932, 1933, 1940) first estimated the effect of plasma-magnetic field interaction

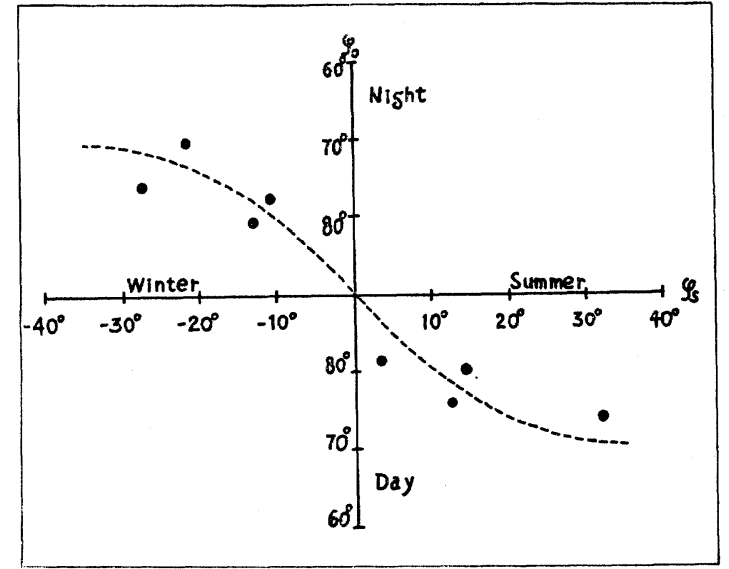

Fig. 3. The relation between the latitude $\varphi_{0}$ of the $D s t$ (SC)-axis and that of the subsolar point $\varphi_{s}$, in geomagnetic coordinates.

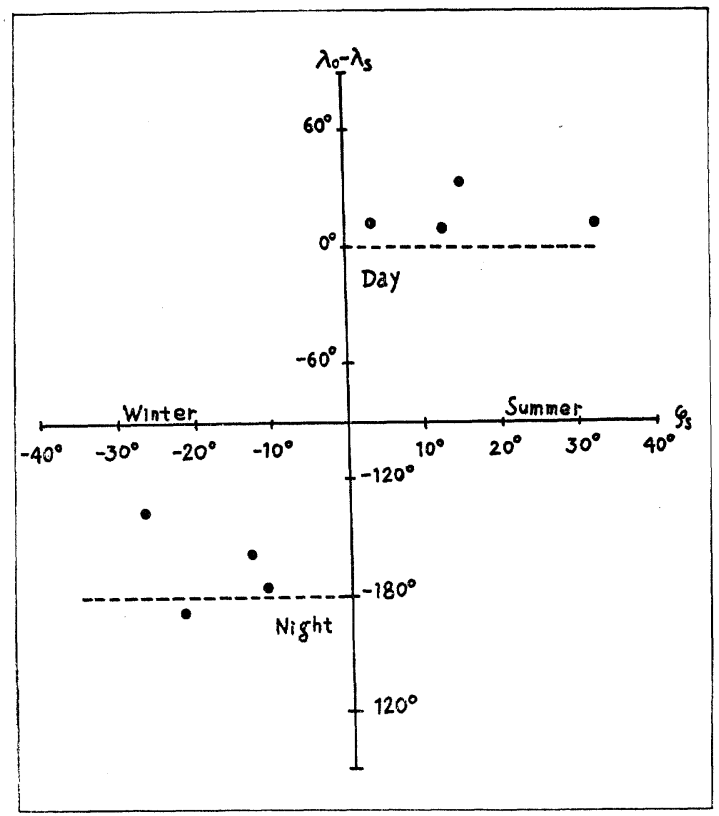

Fig. 4. The relation between the subsolar-point latitude and the longitudinal difference $\left(\lambda_{0}-\lambda_{s}\right)$ of the $\operatorname{Dst}(S C)$-axis and the subsolar point, in geomagnetic coordinates.

by a rough approximation that the front surface of the solar stream is a flat plane parallel to the earth's dipole axis. The magnetic effect on the earth of the approaching solar stream may be represented by the magnetic field of an image dipole on the sun-earth line in the solar stream. Although the front surface of the solar plasma stream is curved so as to form the geomagnetic cavity around the earth, the magnetic field on the earth caused by the solar stream can still be approximated by the field produced by an image dipole on the sun-earth 
line, when the solar stream approaches the earth perpendicularly to the geomagnetic dipole axis. When the solar streamis not perpendicular to the earth's dipole, the geomagnetic cavity boundary is almost spherical, also in this case, at least in low latitudes, as calculated by Spreiter and Briggs (1962), and others. Therefore, the SC-field on the earth's surface may be approximated, even in this case, by the effect of an image dipole on the sun-earth line, although the magnetic moment fo the image dipole may differ from that of the earth, because of the curved form of the geomagnetic cavity.

In the meridional cross-section containing the earth's dipole axis and the sun-earth line, the geometrical relation of the geomagnetic dipole and its image is such as given by Fig. 5 , when the solar declination is $\varphi_{s}$, at the time of the northern winter season. The image dipole is

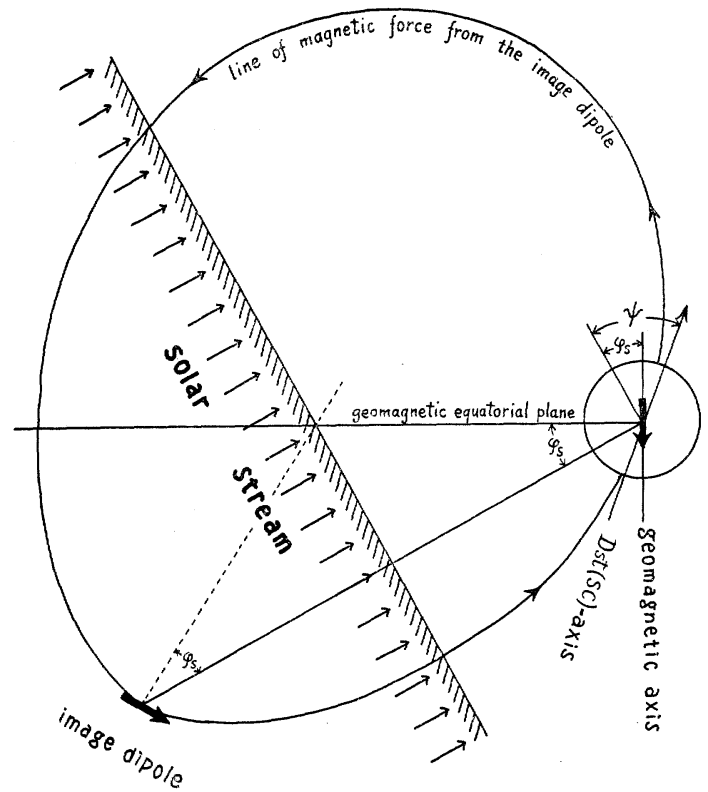

Fig. 5. Geometrical configuration of the earth's dipole and its image in the solar stream in the meridional plane containing the geomagnetic axis and the sun-earth line.

situated at $\left(r^{\prime}, \frac{\pi}{2}+\varphi_{s}, \lambda_{s}\right)$ referred to geomagnetic polar coordinates. The moment of the image dipole is $M^{\prime}$, and the angle between the geomagnetic dipole and its image is $2 \varphi_{s}$. From the geometrical configuration for the lines of magnetic force from the image dipole,

$$
\begin{aligned}
& \tan \psi=2 \tan \varphi_{s}, \\
& \varphi_{0}^{*}=\pi-\phi-\left(\frac{\pi}{2}-\varphi_{s}\right)=\frac{\pi}{2}-\psi+\varphi_{s}, \\
& \therefore \quad \varphi_{0}^{*}=90^{\circ}-\tan ^{-1}\left(2 \tan \varphi_{s}\right)+\varphi_{s},
\end{aligned}
$$

where $\varphi_{0} *$ is the geomagnetic latitude of the $\operatorname{Dst}(S C)$-axis, and this value is independent of $r^{\prime}$ and $M^{\prime}$. The dotted line in Fig. 3 is the calculated value of $\varphi_{0} *$ for a range of $0 \leqq\left|\varphi_{s}\right| \leqq 35^{\circ}$. It is shown that the minimum value of $\varphi_{0}{ }^{*}$ is $70^{\circ} 36^{\prime}$ corresponding to $\left|\varphi_{s}\right|=35^{\circ} 18^{\prime}$, which 
means that the $D s t(S C)$-axis does not tilt away from the geomagnetic dipole axis more than $20^{\circ}$ even in the solstice.

\section{Summary and Discussion}

It is shown in the present paper that the uniform external magnetic field corresponding to $\mathrm{SC}$ in the middle and low latitudes is not always parallel to the geomagnetic dipole axis, but its direction varies systematically, namely, the $\operatorname{Dst}(S C)$-axis inclinces towards the sunlit side in the summer hemisphere and towards the night side in the winter region. The maximum tilt angle is about $20^{\circ}$ even in the solstice.

This systematic seasonal dependence of the $\operatorname{Dst}(S C)$-field in the middle and low latitudes seems to be explained by the interaction with the geomagnetic field of the solar stream, the incident direction of which, with respect to the earth's dipole axis, systematically varies with season. In this simple consideration, the effects of the electromagnetic screening of the ionosphere, and the induction within the earth at the time of SC are not considered. Even when these effects are taken into account, the general conclusion on the seasonal variation in the external $D s t(S C)$-field described in this paper, will remain unchanged. It will be important to check, whether the tendency of seasonal $D s t(S C)$-field variation pointed out in this report is revealed in the seasonal dependence of the diurnal variation in SC-vectors in the middle and low latitudes, especially in the east-west component of the geomagnetic field-change. However, there are too few papers at present on the statistical study of this morphological problem, which are to be compared with the present result.

In concluding, the writers wish to express their hearty thanks to all the magnetic observatoris, which have sent their data to the IGY-WDC. The authors are grateful to Prof. T. Rikitake of the Earthquake Research Institute, and to our colleagues in the Geophysical Institute, University of Tokyo, for their useful comments and suggestions.

\section{References}

Chapman, S., and V.C.A. Ferraro, A new theory of magnetic storms, Terr. Mag., 36, 77-97, 171-186, $1931 ; 37,147-156,421-429,1932 ; 38,79-96,1933$.

Chapman, S., and V.C.A. Ferraro, The theory of the first phase of a geomagnetic storm, Terr. Mag., 45, 245-268, 1940.

Maeda, R., T. Rikitake and T. Nagata, Sudden commencements of geomagnetic storms and their local irregularities, J. Geomag. Geoelectr., 17, in the press, 1965.

Matsushita, S., On geomagnetic sudden commencements, sudden impulses, and storm durations, J. Geophys. Res., 67, 3753-3777, 1962.

Nagata, T., Distribution of SC* of magnetic storms, Rep. Ionos. Res. Japan, 6, 13-30, 1952.

Nagata, T., and S. Abe, Notes on the distribution of SC* in high latitudes, Rep. Ionos. Res. Japan, 9, 39-44, 1955.

Obayashi, T., and J.A. Jacobs, Sudden commencements of magnetic storms and atmospheric dynamo action, J. Geophys. Res., 62, 589-616, 1957.

Oguti, T., Notes on the morphology of SC, Rep. Ionos. Res. Japan, 10, 81-90, 1956.

Sano, Y., Morphological studies on sudden commencements of magnetic storms using the rapid-run magnetograms during the IGY, J. Geomag. Geoelectr., 14, 1-15, 1962.

Sato, T., Sudden commencements of geomagnetic storms in high latitudes, Rep. Ionos. Space Res. Japan, 
15, 215-234, 1961.

Sato, T., Structures of sudden commencements of geomagnetic storms and giant pulsations in high latitudes, Rep. Ionos. Space Res. Japan, 16, 295-333, 1962.

Spreiter, J.R., and B.R. Briggs, Theoretical determination of the form of the boundary of the solar corpuscular stream produced by interaction with the magnetic dipole field of the earth, J. Geophys. Res., 67, 37-51, 1962.

Sugiura, M., The solar diurnal variation in the amplitude of sudden commencements of magnetic storms at the geomagnetic equator, J. Geophys. Res., 58, 558-559, 1953. 